\title{
Phase IQC for the Hierarchical Performance Analysis of Uncertain Large Scale Systems
}

\author{
Khaled Laib, Anton Korniienko, Gérard Scorletti and Florent Morel
}

\begin{abstract}
This paper investigates the performance analysis of uncertain large scale systems. Due to their complexity, the usual robustness analysis methods based on e.g; $\mu$ or Integral Quadratic Constraints cannot be practically applied. In order to address this problem, in [1], we propose to represent a large scale system as an interconnection of sub-systems and to perform a hierarchical analysis by propagating the IQC characterization of each uncertain sub system through the interconnection. For a given computational time, the conservatism of the analysis dramatically depends on the class of IQC under consideration. In this paper, we propose a new class of IQC which characterizes the phase of uncertain system. An application to the robustness analysis of a PLL network reveal that the use of this class of IQC improves the trade-off between conservatism and computation time.
\end{abstract}

Index Terms-Uncertain large scale systems, phase uncertainty, cone sector, hierarchical approach

\section{INTRODUCTION}

Large scale systems (LSS) such as networks or interconnected systems have become important nowadays. With the technological development and the miniaturization of components, high complexity systems are designed in order to achieve a high level of performance, see e.g. Phase Locked Loop (PLL) networks in synchronous multi-core microprocessor systems [2], [3]. However, during the fabrication process, technological dispersions, system ageing, etc. could dramatically affect the performance level: so it is crucial to a priori ensure that the desired level of performance is obtained when the system is realized. Since the first step of the design is to obtain a mathematical model of the system, the differences between the realized system and the model can be expressed as an uncertain model. Ensuring a certain level of performance then reduces to a worst case performance analysis problem (robustness analysis). Robustness analysis investigates the stability and the performance of uncertain Linear Time Invariant (LTI) models. Within this framework, even if the underlying problem is NP hard, many efficient methods were developed based on relaxations as convex optimization problem under Linear Matrix Inequality (LMI) constraints [4], see e.g. the $\mu$ upper bound [5] in the $\mu$ analysis approach [6] or the Integral Quadratic Constraint (IQC) approach [7].

Nevertheless, these methods can not be practically applied to uncertain large scale systems, since the computation time of the robustness analysis becomes dramatically important.

The authors are with Laboratoire Ampère Dpt. EEA of Ecole Centrale de Lyon, Université de Lyon, 69134 Ecully Cedex, France
In order to avoid the direct $\mu$-analysis approach and to reduce the computational load, many techniques are used depending on the nature of the interconnection topology: normal [8] or unitarily diagonalized [9] and [10] (with a normal adjacency matrix). In these works, the authors exploited the particular structure of the interconnection topology and IQC characterization of sub-systems and/or interconnections to derive scalable robust stability conditions. In [11], the authors propose scalable stability test based on Nyquistlike conditions. However, it could be applied only for SISO interconnected systems. An interesting approach is proposed in [12] and [13] where the authors exploit the sparse structure of the interconnection. Based on an IQC characterization of the interconnection, a sparse frequency depended LMI condition is obtained ensuring robust global stability. This condition can be then solved efficiently based on Cholesky factorization techniques [14] assuming a chordal patterns for interconnection topology. However, it could be difficult, for a given LSS, to model it with an interconnection which has a chordal pattern especially if, besides the stability, global system performance is under consideration.

In this paper we rather propose an alternative to [12] and [13] approach for robust performance analysis of LSS without any assumption on the interconnection topology: Hierarchical approach. Initially introduced by Safonov [16], it exploits the hierarchical structure of the interconnection and splits the overall analysis problem into several low dimensional problems. The coupling between these problems is ensured by appropriate IQC conditions such that it implies overall system robust analysis result including stability and performance.

According to the hierarchical approach, a large scale system is represented as a tree with leafs (an interconnection of $N$ systems). Each system $j$ can be described as the interconnection of sub-systems which are the leafs of system $j$ and so on until having sub-systems that can only be described as the certain interconnection of parametric or dynamical uncertainties. Since the effect of an uncertainty and a level of (e.g. $H_{\infty}$ ) performance can be expressed as Integral Quadratic Constraints on input-output signals, the hierarchical approach consists on the recursive propagation of the IQC of the uncertainties to the IQC which defines the performance of the large scale system i.e. propagate the local input-output behaviour evaluated using IQC through the network layer by layer, see [1], [15] for the details. Even if the hierarchical robustness analysis is possibly more conservative than the usual one, the benefit is to reduce the computational time. Furthermore, in order to perform the 
propagation, it is necessary to compute a set of IQCs satisfied by the input and output of an interconnection whose subsystems are defined by a set of IQCs. A set of IQCs is generated by combining elementary classes of IQC. In [1], [15], we investigate the computation of different classes of IQC. Nevertheless, these classes were not adapted for describing (uncertain) phase which is crucial e.g. in vibration control applications. In this paper, in order to improve the trade-off between the conservatism and the computation time of the hierarchical approach, we investigate the computation of an IQC corresponding to the phase information of an interconnection of sub-systems. If the phase of a Single Input Single Output (SISO) system can be easily defined, its definition for a Multiple Input Multiple Output (MIMO) system is more difficult. To address this problem, researchers had defined many concepts such as principal phases [17], phase spread [18], phase matching [19], multi-variable phase margin [20], phase envelope [21], phase sensitive structured value [22] and structured phase margins [23].

In this paper, we reveal that the definition based on the numerical range of a complex matrix $\Gamma$ [18] is a nice candidate to evaluate the uncertain phase since it can be expressed as a quadratic constraint on $z$ and $w$ with $z=\Gamma w$. In [22], the authors considered that each uncertainty block can be phase characterized inside a cone sector and then investigate the stability according to those phase information. The uncertainty phase characterization of [22] can be seen as a special case of the phase characterization presented in this paper in the sense that for the phase rotation a matrix is used rather than a scalar in [22]. The advantage of this rotation matrix is to allow to characterize the phase uncertainty according to any point in the complex plane and not just the origin as in [22]. In this paper, the problem considered is different and more challenging than the robust stability analysis with phase information considered in [22].

In the case of SISO transfer function, the proposed definition reduces to the usual one. Furthermore, using the separation of graph theorem [24], we reveal that an IQC corresponding to the phase information of an interconnection of sub-systems can be computed using quasiconvex optimization involving LMI constraints. We then use the proposed IQC in order to reduce the conservatism of the hierarchical analysis of a PLL network.

This paper is organized as follows: section II presents the problem formulation of the uncertain phase characterization. Some preliminary background is presented followed by the proposed approach in Section III. The main results are presented in Section IV with some illustrative examples in Section V. The advantage of using the phase uncertainty to perform the hierarchical analysis on a PLL network is illustrated in Section VI.

Notations $\mathbb{R H}_{\infty}^{n \times m}$ denotes the set of matrices rational transfer functions with $m$ inputs and $n$ outputs. $A^{*}$ (respectively $A^{T}$ ) is the complex conjugate (respectively transpose) of a the matrix $A . \operatorname{Re}(A)$ (respectively $\operatorname{Re}(x)$ ) represents the real part of a complex matrix $A$ (respectively the complex vector $x$ ) and $\operatorname{Im}(A)$ (respectively $\operatorname{Im}(x)$ ) represents the imaginary part. To simplify the notations, $A^{\mathbb{R}}$ (respectively $x^{\mathbb{R}}$ ) will be used to denote the real part and $A^{\mathbb{I}}$ (respectively $x^{\mathbb{I}}$ ) for the imaginary part. $I_{n}$ and $0_{n \times n}$ is the identity and the zero matrices respectively, when their dimensions are not specified, it is assumed they are know from the context. The $\star$ denotes the Redheffer star product [25]. $\operatorname{bdiag}(A, B, \ldots)$ denotes the block diagonal matrix whose diagonal blocks are $A, B, \ldots$.

\section{PROBLEM Formulation}

Let be the uncertain system $\mathcal{G}=\{M \star \Delta \mid \Delta \in \underline{\Delta}\}$, that is, an uncertain system is represented as the interconnection of a certain part $M \in \mathbb{R} \mathbb{H}_{\infty}^{\left(n_{z}+n_{q}\right) \times\left(n_{w}+n_{p}\right)}$ and an uncertain part $\Delta \in \underline{\Delta}$ where $\underline{\Delta}$ denotes the set of uncertainties traditional in robust analysis literature:

$\underline{\Delta}=\left\{\begin{array}{l|l}\|\Delta\|_{\infty}<1 \\ \Delta=\operatorname{bdiag}\left(\delta_{1}^{r} I_{n_{1}}, \ldots, \delta_{n_{r}}^{r} I_{n_{n_{r}}},\right. \\ \left.\quad \delta_{1}^{c} I_{c_{1}}, \ldots, \delta_{n_{c}}^{c} I_{c_{n_{c}}}, \Delta_{1}, \ldots, \Delta_{n_{f}}\right)\end{array}\right\}$

where

- $\delta_{j}^{r} \in \mathbb{R}$ is a real $n_{j}$ times repeated uncertainty

- $\delta_{j}^{c} \in \mathbb{C}$ is a complex $n_{c}$ times repeated uncertainty

- $\Delta_{j} \in \mathbb{C}^{k_{l}^{j} \times k_{m}^{j}}$ is a a full bloc of complex uncertainties. see Fig. 1. In the sequel, for the sake of briefness, the uncertain system is denoted $M \star \Delta$. Furthermore, for a given frequency $\omega_{0}$, let us denote $\mathcal{G}_{\omega_{0}}$ the set $\left\{M\left(j \omega_{0}\right) \star \Delta\left(j \omega_{0}\right) \mid \Delta \in \underline{\Delta}\right\}$.

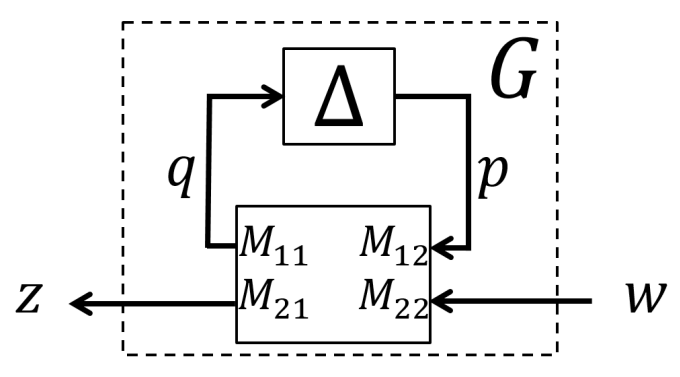

Fig. 1. Uncertain linear system

The numerical range of a complex matrix $\Gamma$, denoted $\mathcal{N}(\Gamma)$, can be used to define the phase of MIMO systems. It is defined to be a compact and convex set of $\mathbb{C}$ given by [18]:

$$
\mathcal{N}(\Gamma)=\left\{w^{*} z \mid z=\Gamma w, w \in \mathbb{C}^{n_{w}} \text { and }\|w\|=1\right\}
$$

In the case of a MIMO system $G, \Gamma$ represents the frequency response of $G$ at the frequency $\omega_{0}$. In order to define the phase of an uncertain MIMO system $M \star \Delta$, the numerical range is extended to the union of the numerical ranges $\mathcal{N}\left(G\left(j \omega_{0}\right)\right)$ for any $G \in \mathcal{G}$ which will be referred to as union of numerical ranges. Let us define in the complex plane the cone sector as the sector containing all these numerical ranges. It is defined by a spread angle $\alpha$ and the angle $\gamma$ measured between the bisectrix of $\alpha$ and the real axe direction, see Fig. II where a sampling of the union of 
numerical ranges, for a given frequency $\omega_{0}$, and the cone sector with a centre at the origin are represented. For any

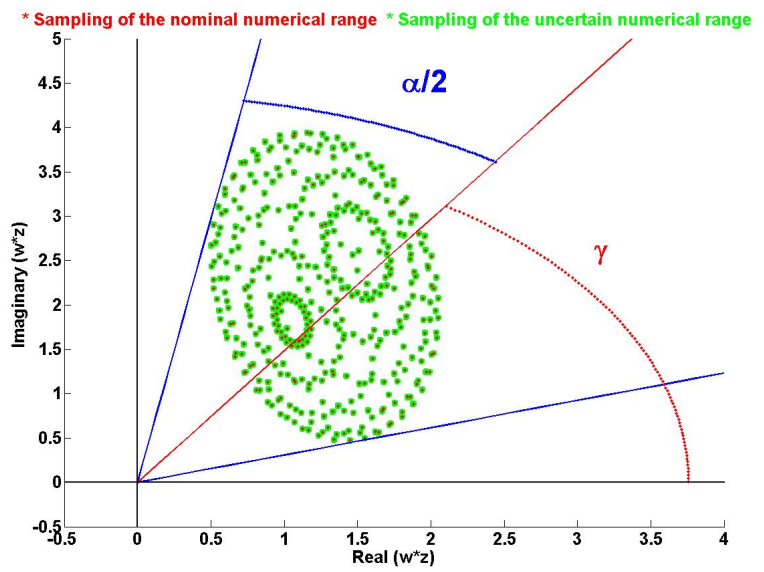

Fig. 2. Cone sector containing at a frequency $\omega_{0}, \mathcal{N}\left(G\left(j \omega_{0}\right)\right.$ for any $G \in \mathcal{G}$

numerical range $\mathcal{N}(\Gamma)$ which is not contained in the right half plane, one can make $\mathcal{N}(\Gamma)$ rotated by an angle $-\gamma$ such that the resulting numerical range $\mathcal{N}\left(e^{-j \gamma} \Gamma\right)$ will be centred around the real axis in the right half plane with a new $\tilde{\gamma}=0$. To improve the flexibility of the results, this rotation $e^{-j \gamma}$ can be generalized to be an homothetic transformation and a rotation using a scaling matrix $\Omega \in \mathbb{C}^{n_{z} \times n_{w}}$. The resulting numerical range is $\mathcal{N}\left(\Omega^{*} \Gamma\right)$ and it belongs to the sector centred at the origin with an angle spread $\alpha$ denoted $\sec (0, \alpha)$. This scaling matrix is a generalization of the rotation introduced in [22]. The uncertain phase problem can then be formulated as follows.

Problem 2.1: Let $\mathcal{G}$ be an uncertain system. For a given frequency $\omega_{0}$, find the smallest $\alpha$ such that:

$$
\exists \Omega \in \mathbb{C}^{n_{z} \times n_{w}}, \forall \Gamma \in \mathcal{G}_{\omega_{0}}, \mathcal{N}\left(\Omega^{*} \Gamma\right) \subset \sec (0, \alpha) .
$$

Remark 2.1: For SISO LTI systems without uncertainty, the numerical range reduces to one point $\mathcal{N}(\Gamma)=G\left(j \omega_{0}\right)$; in this case $\alpha=0$ and $\Omega=e^{j \arg \left(G\left(j \omega_{0}\right)\right)}$.

In the general case, one can define an offset characterized by $C \in \mathbb{C}^{n_{z} \times n_{w}}$ and seek the smallest sector with the corresponding notation $\sec (C, \alpha)$. This problem can be solved by finding the cone sector containing all the numerical ranges of $\Gamma-C$. Hence, one can search for the smallest $\alpha$ such that the numerical range $\mathcal{N}\left(\Omega^{*}(\Gamma-C)\right)$ will be on the right half plane and centred at the origin.

Problem 2.2: Let $\mathcal{G}$ be an uncertain system. For a given frequency $\omega_{0}$ and a given offset $C$, find the smallest $\alpha$ such that:

$$
\exists \Omega \in \mathbb{C}^{n_{z} \times n_{w}}, \forall \Gamma \in \mathcal{G}_{\omega_{0}}, \mathcal{N}\left(\Omega^{*}(\Gamma-C)\right) \subset \sec (0, \alpha) .
$$

\section{PREliminary AND PROPOSED APPROACH}

\section{A. Preliminary}

Definition 3.1: The stable system $G$ is said to be $\{X(j \omega), Y(j \omega), Z(j \omega)\} \quad$ dissipative, with $X(j \omega)=$
$X^{*}(j \omega) \in \mathbb{C}^{n_{z} \times n_{z}}, Y(j \omega) \in \mathbb{C}^{n_{z} \times n_{w}}$ and $Z(j \omega)=$ $Z^{*}(j \omega) \in \mathbb{C}^{n_{w} \times n_{w}}$ if for every $z(j \omega)$ and $w(j \omega)$ such that $z(j \omega)=G(j \omega) w(j \omega)$ :

$$
\left(\begin{array}{c}
z(j \omega) \\
w(j \omega)
\end{array}\right)^{*}\left(\begin{array}{cc}
X(j \omega) & Y(j \omega) \\
Y(j \omega)^{*} & Z(j \omega)
\end{array}\right)\left(\begin{array}{c}
z(j \omega) \\
w(j \omega)
\end{array}\right)<0
$$

Remark 3.1: Dissipativity properties define a set of relations describing the input-output behaviour of a system $G$. They represent a set of Quadratic Constraints (QC) involving the input-output signals in the case of LTI systems. In a more general framework for non linear systems, they can be generalized to a set of Integral Quadratic Constraints (IQC) [7]. Note that in the case of LTI systems, IQC are simplified to $\mathrm{QC}$.

Without lost of generality, the frequency dependence will be dropped in the sequel. A frequency griding is defined and the different operations and ideas will be introduced for a given frequency $\omega_{0}$ i.e. the $\left\{X\left(j \omega_{0}\right), Y\left(j \omega_{0}\right), Z\left(j \omega_{0}\right)\right\}$ dissipativity will be written as:

$$
\left(\begin{array}{c}
z \\
w
\end{array}\right)^{*}\left(\begin{array}{cc}
X & Y \\
Y^{*} & Z
\end{array}\right)\left(\begin{array}{c}
z \\
w
\end{array}\right)<0
$$

The following result is a direct application of a Theorem 4.1 of [24].

Theorem 3.1: The uncertain system $M \star \Delta$ is $\{X, Y, Z\}$ dissipative for every $\Delta \in \underline{\Delta}$ if and only if there exists an hermitian matrix $\Phi=\Phi^{*}$ such that:

1)

$$
\left(\begin{array}{c}
\Delta \\
I
\end{array}\right)^{*} \underbrace{\left(\begin{array}{ll}
\Phi_{11} & \Phi_{12} \\
\Phi_{12}^{*} & \Phi_{22}
\end{array}\right)}_{\Phi}\left(\begin{array}{c}
\Delta \\
I
\end{array}\right)>0 \quad \forall \Delta \in \underline{\Delta}
$$

and

2)

$$
\left(\begin{array}{c}
M \\
I
\end{array}\right)^{*}\left(\begin{array}{llll}
\Phi_{22} & 0 & \Phi_{12}^{*} & 0 \\
0 & X & 0 & Y \\
\Phi_{12} & 0 & \Phi_{11} & 0 \\
0 & Y^{*} & 0 & Z
\end{array}\right)\left(\begin{array}{c}
M \\
I
\end{array}\right)<0
$$

Remark 3.2: Theorem 3.1 presents necessary and sufficient conditions for the uncertain system $M \star \Delta$ to be $\{X, Y, Z\}$ dissipative. Testing these conditions can be expressed as the optimization problem of finding $\Phi$ such that conditions 1) and 2) are satisfied. However, condition 1) makes the optimization problem infinite dimensional since it has to be tested for all $\Delta \in \underline{\Delta}$, which is difficult from a computational point of view. The complexity can be reduced by introducing an affine set $\boldsymbol{\Phi}_{\boldsymbol{\Delta}}$ such that for any $\Phi \in \boldsymbol{\Phi}_{\boldsymbol{\Delta}}$, condition 1) is satisfied. The set $\boldsymbol{\Phi}_{\boldsymbol{\Delta}}$ depends on the nature of $\Delta$. In this case, Theorem 3.1 gives only sufficient conditions for all $\Delta \in \Delta$ and the conditions of Theorem 3.1 define a finite dimensional LMI optimization problem that consists in finding one $\Phi \in \boldsymbol{\Phi}_{\boldsymbol{\Delta}}$ such that the second condition of Theorem 3.1 is satisfied. From a computational point of view, this problem can be efficiently solved. The consequence of this parametrization of $\Phi$ is a possible conservatism in the obtained results. However, this last can be reduced by an 
appropriate choice of $\boldsymbol{\Phi}_{\boldsymbol{\Delta}}$ depending on the class of the uncertainties $\underline{\Delta}$.

Remark 3.3: If conditions 1) and 2) of Theorem 3.1 are satisfied for all $\omega$ and since $\Delta$ is a connected set that contains 0 then the stability is guaranteed, see Theorem 4.1 of [24]. Furthermore, when $\Delta$ is not a ball of center 0 , conditions 1) and 2) still imply the results of Theorem 3.1 if $\boldsymbol{\Delta}$ is a connected set with $\Delta_{0}$ such that $M \star \Delta_{0}$ is stable.

\section{B. Proposed approach}

Find a cone sector $\sec (0, \alpha)$ of Problem 2.1 (that contains $\left.\mathcal{N}(\Gamma), \forall \Gamma \in \mathcal{G}_{j \omega_{0}}\right)$ can be formulated as find $b$ such that:

$$
\begin{aligned}
& \operatorname{Re}\left(w^{*} \Omega^{*} z\right)-\beta \operatorname{Im}\left(w^{*} \Omega^{*} z\right)>0 \quad \beta= \pm b \\
& \forall z, w \quad \text { such that } \exists \Delta \in \underline{\Delta} \text { and } z=M \star \Delta w
\end{aligned}
$$

$b>0$ defines a slope of two lines forming the cone sector and is related to $\alpha$ by $\tan \left(\frac{\alpha}{2}\right)=\frac{1}{b}$. Please note that the matrix $\Omega$ is used to rotate all numerical ranges in the right half plane such that $\gamma=0$, see Fig. 3 . Inequalities (4) represent a couple of Quadratic Constraints

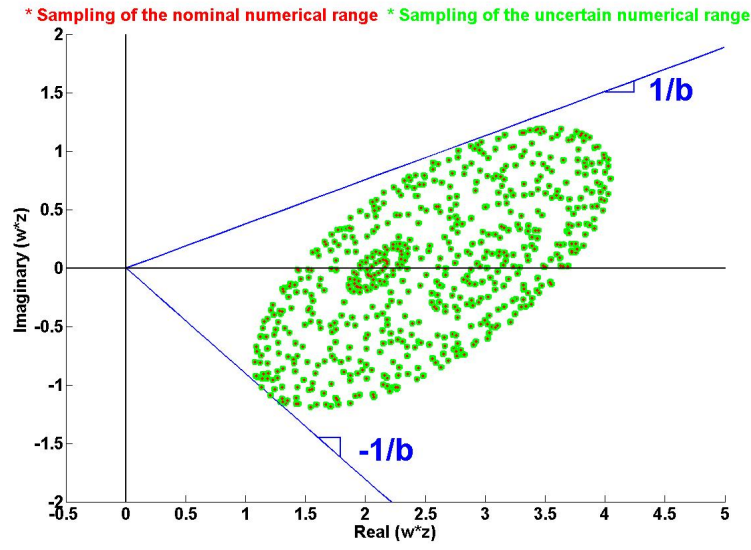

Fig. 3. Illustration of $\mathcal{N}(\Gamma)$ and the cone sector $\sec (0, \alpha)$

(QC) characterizing the input output behaviour of the system $G$ with its input and output signals $w$ and $z$. The cone sector can hence be formulated as:

$$
\left(\begin{array}{c}
z \\
w
\end{array}\right)^{*}\left(\begin{array}{cc}
0 & \Omega(-I+j \beta I) \\
(\Omega(-I+j \beta I))^{*} & 0
\end{array}\right)\left(\begin{array}{c}
z \\
w
\end{array}\right)<0
$$

For any other given $C \neq 0$ i.e. as defined in Problem 2.2, the cone sector $\sec (C, \alpha)$ is given by:

$$
\left(\begin{array}{c}
z \\
w
\end{array}\right)^{*}\left(\begin{array}{cc}
X & Y \\
Y^{*} & Z
\end{array}\right)\left(\begin{array}{c}
z \\
w
\end{array}\right)<0
$$

Where:

$$
X=0 Y=\Omega(-I+j \beta I) Z=-\left(Y^{*} C+C^{*} Y\right)
$$

With $\beta= \pm b$, the inequalities of (6) define two dissipativity properties $\left\{X_{1}, Y_{1}, Z_{1}\right\}$ for $\beta=+b$ and $\left\{X_{2}, Y_{2}, Z_{2}\right\}$ for $\beta=-b$.

\section{MAIN RESULTS}

Theorem 4.1: For a given $C$, given $\alpha$ and for the uncertain system $M \star \Delta$. If there exist: $\Omega,\left(\Phi_{111}, \Phi_{121}, \Phi_{221}\right) \in \boldsymbol{\Phi}_{\boldsymbol{\Delta}}$ and $\left(\Phi_{112}, \Phi_{122}, \Phi_{222}\right) \in \boldsymbol{\Phi}_{\boldsymbol{\Delta}}$ such that:

$$
\left(\begin{array}{c}
M \\
I
\end{array}\right)^{*}\left(\begin{array}{cccc}
\Phi_{22 i} & 0 & \Phi_{12 i}^{*} & 0 \\
0 & X_{i} & 0 & Y_{i} \\
\Phi_{12 i} & 0 & \Phi_{11 i} & 0 \\
0 & Y_{i}^{*} & 0 & Z_{i}
\end{array}\right)\left(\begin{array}{c}
M \\
I
\end{array}\right)<0 \quad i=1,2
$$

Where:

$$
\begin{aligned}
& X_{1}=0, X_{2}=0 \\
& Y_{1}=\Omega\left(-I+j \cot \left(\frac{\alpha}{2}\right) I\right), \quad Z_{1}=-\left(Y_{1}^{*} C+C^{*} Y_{1}\right) \\
& Y_{2}=\Omega\left(-I-j \cot \left(\frac{\alpha}{2}\right) I\right), \quad Z_{2}=-\left(Y_{2}^{*} C+C^{*} Y_{2}\right)
\end{aligned}
$$

Then, the cone sector $\sec (0, \alpha)$ contains all the numerical ranges of the uncertain system $M \star \Delta-C$ scaled by $\Omega$ :

$$
\mathcal{N}\left(\Omega^{*}(M \star \Delta-C)\right) \subset \sec (0, \alpha) \quad \forall \Delta \in \underline{\Delta}
$$

Proof: Theorem 4.1 is a direct application of Theorem 3.1, with $\{X, Y, Z\}$ dissipativity (6) for $b=\cot \left(\frac{\alpha}{2}\right)$ and by choosing some particular structure defined by $\Phi_{\Delta}$, see Remark 3.2.

Theorem 4.1 defines an optimization problem. The decision variables are: $\Omega, \Phi_{111}, \Phi_{121}, \Phi_{221}, \Phi_{112}, \Phi_{122}$, and $\Phi_{222}$. It proposes only sufficient conditions for the existence of a cone sector $\sec (0, \alpha)$ containing all the numerical ranges. Testing them is a finite dimensional feasibility problem involving LMI which can be efficiently solved [26]. If a cone sector exists, it is possible to search for the smallest one. To this purpose, we introduce a size measure for the cone sector. The objective is to minimize $\alpha$. Since $\tan \left(\frac{\alpha}{2}\right)=\frac{1}{b}$ and $b>0$ :

$$
\begin{array}{rlll}
\min _{\alpha} \quad \alpha \quad & \Leftrightarrow \quad \min _{\alpha} \tan (\alpha / 2) \\
& \Leftrightarrow \quad \min 1 / b
\end{array}
$$

Then, $1 / b$ represents a size measure for the cone. Since the conditions of Theorem 4.1 are only sufficient, minimizing $1 / b$ such that they are satisfied implies the minimization of an upper bound $\widetilde{\alpha}$ on the spread angle $\alpha$. Nevertheless, how this upper bound is close to real spread depends on the choice of $\boldsymbol{\Phi}_{\Delta}$, see Remark 3.2.

After defining the size measure of the cone sector $\sec (C, \alpha)$, Problem 2.2 can be addressed such that an upper bound on the angle $\alpha$ can be computed efficiently.

Theorem 4.2: An upper bound on the angle $\alpha$ defined in Problem 2.2 can be obtained by finding $\Omega, \widehat{D}_{1}, \widehat{G}_{1}, \widetilde{D}_{1}$, $\widetilde{G}_{1}, \widehat{D}_{2}, \widehat{G}_{2}, \widetilde{D}_{2}$, and $\widetilde{G}_{2}$ that minimize $\lambda$ in the following Generalized Eigenvalues Problem (GEVP):

$$
\begin{array}{ll}
\min _{\lambda, \Omega} & \lambda \\
\widehat{D}_{1}, \widehat{G}_{1}, \widetilde{D}_{1}, \widetilde{G}_{1} & \\
\widehat{D}_{2}, \widehat{G}_{2}, \widetilde{D}_{2}, \widetilde{G}_{2} &
\end{array}
$$


1)

$$
\begin{aligned}
& \lambda\left(\begin{array}{cc}
\widehat{D}_{1} & 0 \\
0 & \widehat{D}_{2}
\end{array}\right)+\left(\begin{array}{cc}
\widetilde{D}_{1} & 0 \\
0 & -\widetilde{D}_{2}
\end{array}\right)>0 \\
& \lambda\left(\begin{array}{cc}
M & 0 \\
I & 0 \\
0 & M \\
0 & I
\end{array}\right)^{*}\left(\begin{array}{cc}
B_{1} & 0 \\
0 & B_{2}
\end{array}\right)\left(\begin{array}{cc}
M & 0 \\
I & 0 \\
0 & M \\
0 & I
\end{array}\right)+\ldots \\
& \quad \cdots+\left(\begin{array}{cc}
M & 0 \\
I & 0 \\
0 & M \\
0 & I
\end{array}\right)^{*}\left(\begin{array}{cc}
A_{1} & 0 \\
0 & A_{2}
\end{array}\right)\left(\begin{array}{cc}
M & 0 \\
I & 0 \\
0 & M \\
0 & I
\end{array}\right)>0
\end{aligned}
$$

2)

$$
\begin{aligned}
& \left(\begin{array}{cc}
\widehat{D}_{1} & 0 \\
0 & \widehat{D}_{2}
\end{array}\right)>0 \\
& \left(\begin{array}{cc}
M & 0 \\
I & 0 \\
0 & M \\
0 & I
\end{array}\right)^{*}\left(\begin{array}{cc}
B_{1} & 0 \\
0 & B_{2}
\end{array}\right)\left(\begin{array}{cc}
M & 0 \\
I & 0 \\
0 & M \\
0 & I
\end{array}\right)>0
\end{aligned}
$$

with $i=\{1,2\}, B_{i}$ and $A_{i}$ are given by:

$$
\begin{aligned}
B_{i} & =\left(\begin{array}{cccc}
-\widehat{D}_{i} & 0 & -\widehat{G}_{i}^{*} & 0 \\
0 & 0 & 0 & \Omega \\
-\widehat{G}_{i} & 0 & \widetilde{D}_{i} & 0 \\
0 & \Omega^{*} & 0 & -\left(\Omega^{*} C+C^{*} \Omega\right)
\end{array}\right) \\
A_{i} & =(-1)^{i-1}\left(\begin{array}{cccc}
-\widetilde{D}_{i} & 0 & -\widetilde{G}_{i}^{*} & 0 \\
0 & 0 & 0 & -j \Omega \\
-\widetilde{G}_{i} & 0 & \widetilde{D}_{i} & 0 \\
0 & (-j \Omega)^{*} & 0 & -j\left(\Omega^{*} C-C^{*} \Omega\right)
\end{array}\right)
\end{aligned}
$$

Proof: For the cone sector defined by the dissipativity inequality (6) and using Theorem 4.1, Problem 2.2 can be solved with finding a minimum upper bound $\widetilde{\alpha}$ on $\alpha$ such that $\sec (C, \alpha) \subset \sec (C, \widetilde{\alpha})$. Hence, Problem 2.2 becomes: Find $\Phi_{i}=\left\{\Phi_{11 i}, \Phi_{12 i}, \Phi_{22 i}\right\} \in \Phi_{\Delta}, X_{i}, Y_{i}$, and $Z_{i}, i=$ $\{1,2\}$, that maximize $b$ such that:

$$
\left(\begin{array}{c}
M \\
I
\end{array}\right)^{*}\left(\begin{array}{cccc}
-\Phi_{22 i} & 0 & -\Phi_{12 i}^{*} & 0 \\
0 & -X_{i} & 0 & -Y_{i} \\
-\Phi_{12 i} & 0 & -\Phi_{11 i} & 0 \\
0 & -Y_{i}^{*} & 0 & -Z_{i}
\end{array}\right)\left(\begin{array}{c}
M \\
I
\end{array}\right)>0 \quad i=1,2
$$

where $X_{i}, Y_{i}$ and $Z_{i}$ defined as in (6) for $\beta=+b$ and $\beta=-b$. Let us define $\Phi_{1}$ and $\Phi_{2}$ as:

$$
\Phi_{1}=\widehat{\Phi}_{1}+b \widetilde{\Phi}_{1} \quad \Phi_{2}=\widehat{\Phi}_{2}-b \widetilde{\Phi}_{2}
$$

where $\widehat{\Phi}_{i}$ and $\widetilde{\Phi}_{i}$ are chosen in the form of $D G$ scaling presented in [5]:

$$
\widehat{\Phi}_{i}=\left(\begin{array}{cc}
-\widehat{D}_{i} & \widehat{G}_{i} \\
\widehat{G}_{i}^{*} & \widehat{D}_{i}
\end{array}\right) \quad \widetilde{\Phi}_{i}=\left(\begin{array}{cc}
-\widetilde{D}_{i} & \widetilde{G}_{i} \\
\widetilde{G}_{i}^{*} & \widetilde{D}_{i}
\end{array}\right)
$$

with: $\widehat{D}_{i}>0$. In order to make sure that $\Phi_{i} \in \mathbf{\Phi}_{\boldsymbol{\Delta}}$, one needs to guarantee that:

$$
\widehat{D}_{1}+b \widetilde{D}_{1}>0 \quad \widehat{D}_{2}-b \widetilde{D}_{2}>0
$$

Then, inequalities (7) become:

$$
\begin{aligned}
& \left(\begin{array}{c}
M \\
I
\end{array}\right)^{*}\left[\left(\begin{array}{cccc}
-\widehat{D}_{i} & 0 & -\widehat{G}_{i}^{*} & 0 \\
0 & 0 & 0 \\
-\widehat{G}_{i} & 0 & 0 \\
0 & \Omega^{*} & 0 & -\left(\Omega^{*} C+C^{*} \Omega\right)
\end{array}\right)\right. \\
& \left.+(-1)^{i-1} b\left(\begin{array}{cccc}
-\widetilde{D}_{i} & 0 & -\widetilde{G}_{i}^{*} & 0 \\
0 & 0 & 0 & -j \Omega \\
-\widetilde{G}_{i} & 0 & \widetilde{D}_{i} & 0 \\
0 & (-j \Omega)^{*} & 0 & -j\left(\Omega^{*} C-C^{*} \Omega\right)
\end{array}\right)\right]\left(\begin{array}{c}
M \\
I
\end{array}\right)>0
\end{aligned}
$$

Evaluating for $i=\{1,2\}$ and since $b>0$, inequalities (9) and (10) become:

$$
\begin{aligned}
& \frac{1}{b} \widehat{D}_{1}+\widetilde{D}_{1}>0 \\
& \frac{1}{b} \widehat{D}_{2}-\widetilde{D}_{2}>0 \\
& \frac{1}{b}\left(\begin{array}{c}
M \\
I
\end{array}\right)^{*} B_{1}\left(\begin{array}{c}
M \\
I
\end{array}\right)+\left(\begin{array}{c}
M \\
I
\end{array}\right)^{*} A_{1}\left(\begin{array}{c}
M \\
I
\end{array}\right)>0 \\
& \frac{1}{b}\left(\begin{array}{c}
M \\
I
\end{array}\right)^{*} B_{2}\left(\begin{array}{c}
M \\
I
\end{array}\right)+\left(\begin{array}{c}
M \\
I
\end{array}\right)^{*} A_{2}\left(\begin{array}{c}
M \\
I
\end{array}\right)>0
\end{aligned}
$$

The last inequalities can be combined together as it is shown in the first condition of Theorem 4.2. Hence, minimizing $1 / b$ such that the last inequalities holds is a Generalized Eigenvalues Problem since condition 2) of Theorem 4.2 holds.

Remark 4.1: Theorem 4.2 is a Generalized Eigenvalues Problem, it has been proved that it is a quasiconvex optimization problem [26]. It can be solved efficiently using the projective method described in [27]. Nevertheless, since the conditions are sufficient, Theorem 4.2 allows to compute a $\operatorname{sector} \sec (C, \widetilde{\alpha})$ which contains the smallest cone sector $\sec (C, \alpha)$. The conservatism can be reduced by an appropriate choice of $\Phi_{\Delta}$ depending on the nature of uncertainties: $D$ scaling [6], $D G$ scaling [5] or $D G L$ scaling [28]. Here the $D G$ scaling is chosen to characterize $\Phi_{i}$ for illustration purposes. Nevertheless, one can choose other types of scaling depending on the nature of uncertainties. In this case, the structure of parametrization matrices $\Phi_{i}$ will be different but the idea remains the same i.e. decomposing $\Phi_{i}$ into two parts: $\widehat{\Phi}_{i}$ and $\widetilde{\Phi}_{i}$.

Remark 4.2: With respect to Theorem 4.1, Theorem 4.2 introduces an extra condition (condition 2) in order to obtain a Generalized Eigenvalues problem due to the advantage of this optimization problem. This condition has two nice justifications: first, it guarantees that the interconnection $M \star \Delta, \forall \Delta \in \underline{\Delta}$ is stable. Second, it guarantee that $\mathcal{N}\left(\Omega^{*}(\Gamma-C)\right)$ is located in the right half plane. Then, one needs to minimize the upper bound $\widetilde{\alpha}$ :

$$
\widetilde{\alpha} \in\left[-\frac{\pi}{2},+\frac{\pi}{2}\right] \quad \text { s.t } \mathcal{N}\left(\Omega^{*}(\Gamma-C)\right) \subset \sec (0, \widetilde{\alpha})
$$

On the other side, introducing this condition enforces more constraints on $\Phi_{1} \in \boldsymbol{\Phi}_{\boldsymbol{\Delta}}$ and $\Phi_{2} \in \boldsymbol{\Phi}_{\boldsymbol{\Delta}}$ if they are chosen as in (8) with $\widetilde{\Phi}_{i}=0$ : they must satisfy that all the numerical ranges $\mathcal{N}\left(\Omega^{*}(\Gamma-C)\right)$ are in the right half plane and respect their dissipative properties at the same time. Hence, an extra conservatism may appear (besides that of Remark 4.1). To overcome this issue, $\Phi_{1}$ and $\Phi_{2}$ are chosen of the form (8). Here, $\widehat{D}_{i}$ and $\widehat{G}_{i}$ ensure that the interconnection $M \star \Delta$ is stable and $\mathcal{N}\left(\Omega^{*}(\Gamma-C)\right)$ is in the right half plane. While $\widetilde{D}_{i}$ and $\widetilde{G}_{i}$ introduce an additional degree of freedom to ensure that $\mathcal{N}\left(\Omega^{*}(\Gamma-C)\right) \subset \sec (0, \widetilde{\alpha})$. Hence, with this choice, the conservatism is reduced. 


\section{ILLUSTRATION EXAMPLES}

In this section, we will show some examples that illustrate our results graphically.

\section{A. SISO Uncertain Systems}

The following example is inspired from [1]. Let us consider the following SISO example with structured scalar uncertainties:

$$
G^{S I S O}\left(j \omega_{0}\right)=\frac{-\omega_{0}^{2}+2 \xi_{n} \omega_{n}\left(j \omega_{0}\right)+\omega_{n}^{2}}{-\omega_{0}^{2}+2 \xi_{d} \omega_{d}\left(j \omega_{0}\right)+\omega_{d}^{2}}
$$

with $\omega_{0}=1 \mathrm{rad} / \mathrm{s}, \xi_{n}=0.7$ and $\xi_{d}=0.01$. The uncertainties are parametric and they are defined by: $\omega_{n} \in$ $[0.1,0.5]$ and $\omega_{d} \in[9,10]$. The results are illustrated in Fig.4.

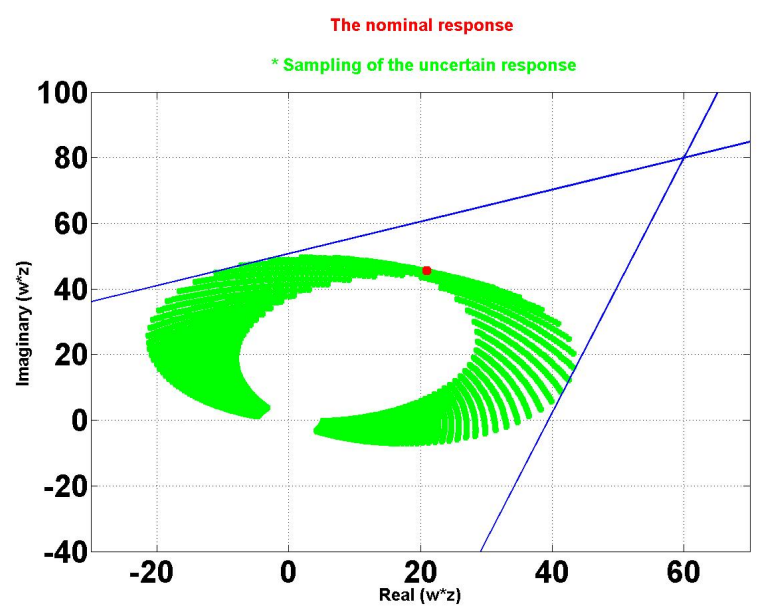

Fig. 4. Visualisation of cone sector for SISO example

The sampling of the uncertain response presented in green dots is obtained for a griding of $\omega_{n}$ and $\omega_{d}$ equal to 0.01 and 0.001 respectively. The nominal response is equal to $21+45 i$ and it is presented with a red dot. The cone offset $\mathrm{C}$ was fixed arbitrary at $C=60+80 i$. The obtained results shows very low conservatism and define a cone angle $\tilde{\alpha}=49.52^{\circ}$.

\section{B. MIMO Uncertain Systems}

For MIMO case, a simple example can be given by:

$$
G^{M I M O}\left(j \omega_{0}\right)=o_{d i r} \times \times G^{S I S O}\left(j \omega_{0}\right) \times i_{d i r}-C
$$

with same frequency $\omega_{0}=1 \mathrm{rad} / \mathrm{s}, o_{\text {dir }}=\left(\begin{array}{ll}1 & -1.5\end{array}\right)^{T}$, $i_{\text {dir }}=\left(\begin{array}{ll}1 & 2\end{array}\right)$ and $C=50(1+5 i) I_{2}$. Fig. 5 shows a sampling of the union of numerical ranges while Fig. 6. shows a sampling of the union of numerical ranges scaled by $\Omega^{*}$ with the obtained cone sector.

As it can be seen in Fig 5 and Fig 6, the numerical range $\Gamma(\mathcal{N}-C)$ which was located in the left half plane was rotated and scaled in size by $\Omega^{*}$. Once it is in the right half plane, a cone sector $\sec (0, \tilde{\alpha})$ is found: $\tilde{\alpha}=25^{\circ}$. See Fig. 6 for illustration purposes, please note that in the imaginary and the real axe scales are not equal.

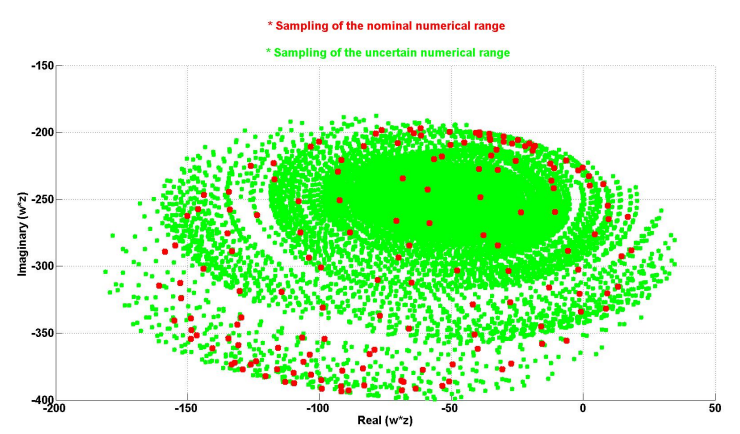

Fig. 5. Visualisation of the nominal and the uncertain numerical ranges

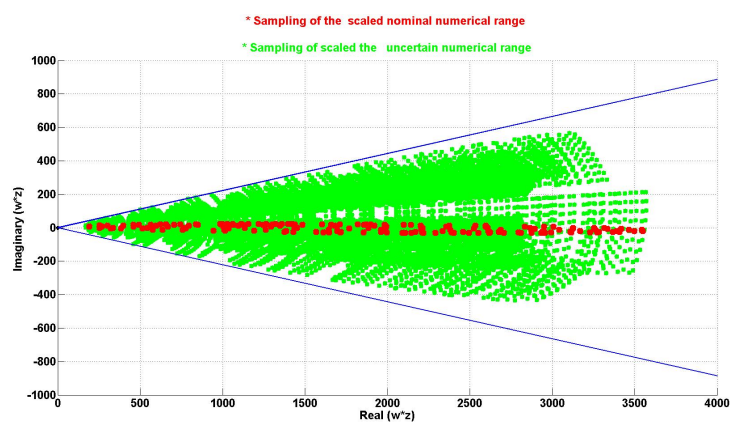

Fig. 6. Visualisation of the scaled nominal and the uncertain numerical ranges

\section{Large Scale System Hierarchical Analysis}

Let us consider the example of the performance analysis of the active clock distribution network of [2]. It is composed of $N=16$ mutually synchronized Phase Locked Loop (PLL) delivering the clock signals to the chip. In order to synchronize all the network, the PLLs exchange information through an interconnection structure. This example is suitable for illustration of the proposed hierarchical analysis approach as the performance is naturally evaluated in the frequency domain [15].

\section{A. PLL network description}

In this application, all the PLLs are homogeneous i.e. have the same description and uncertainty set $\underline{\Delta}$. Due to the manufacturing process, technological dispersions are inevitable. They can be presented as parametric uncertainties belonging to the same set $\underline{\Delta}$. Then, the description of the $N$ PLLs is:

$$
T_{i}\left(j \omega_{0}\right)=\frac{k_{i}\left(j \omega_{0}+a_{i}\right)}{-\omega^{2}+k_{i} j \omega_{0}+k_{i} a_{i}} \quad \forall i \in\{1, \ldots, N\}
$$

Where $k_{i} \in[0.76,6.84] \times 10^{4}, a_{i} \in[91.1,273.3]$ and $\omega_{0}$ is the current frequency defined by griding. Furthermore, $T_{i}\left(j \omega_{0}\right)$ can written as the interconnection of certain and uncertain part:

$$
T_{i}\left(j \omega_{0}\right)=\Delta_{i} \star M_{P L L} \quad \Delta_{i} \in \underline{\Delta}
$$

With $\Delta_{i}$ is given by:

$$
\underline{\Delta}=\left\{\Delta_{i}=\left(\begin{array}{cc}
\delta_{k_{i}} & 0 \\
0 & \delta_{a_{i}}
\end{array}\right) \quad\left\|\Delta_{i}\right\|_{\infty} \leq 1\right\}
$$


The exchange of information between PLLs is modelled by an interconnection matrix defined in [1] (see equation (13)). The performance of this network can be characterized by its global input and output $w_{g}$ and $z_{g}$ using the global frequency response magnitude bound. (See more details in [1] and [15]).

\section{B. Hierarchical Approach}

For the PLL network, the hierarchical approach consists of two steps:

1) Local step: Characterize the input-output behaviour of each PLL using dissipativity properties. In addition to the cone sector, one can use other quadratic constraints that can be interpreted by simple geometric forms: disc and band, see [1] and [15]) for more details. Since all the PLLs are homogeneous, the dissipativity properties obtained for one PLL are valid for all the 16 PLL.

2) Global step: Evaluate the performance of the network by finding a minimal upper bound on its frequency response magnitude using the dissipativity properties describing the PLLs and obtained in the local step. See [16], [1] and [15] for more details. In this step, one can combine the different dissipativity properties of each PLL: disc, disc+band, disc+cone, etc. and propagate this input-output characterization of each sub-system to investigate the performance of the network.

\section{The PLL network performance analysis}

The performance analysis results of the PLL network presented in Fig. 7 and summarized in TABLE I. The direct

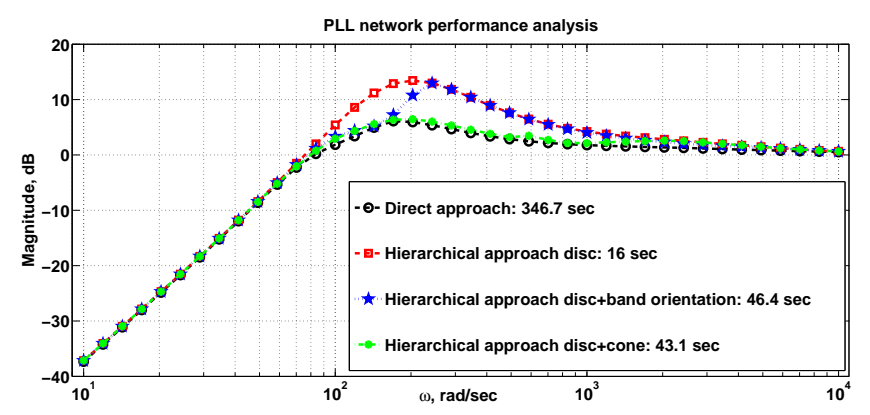

Fig. 7. The PLL network performance analysis

$\mu$-analysis approach presents the less conservative results with a maximum peak of $6.01 d B$ comparing to the different hierarchical approaches. However, computation time is significant: $346.7 \mathrm{~s}$.

\begin{tabular}{|c|c|c|}
\hline Approach & Maximum peak & Computation time \\
\hline$\mu$-analysis & $6.01 d B$ & $347.6 \mathrm{~s}$ \\
\hline Hierarchical: disc & $13.44 d B$ & $16 \mathrm{~s}$ \\
\hline Hierarchical: disc+band & $12.97 d B$ & $46.4 \mathrm{~s}$ \\
\hline Hierarchical: disc+cone & $6.45 d B$ & $43.1 \mathrm{~s}$ \\
\hline
\end{tabular}

TABLE I

COMPARISON BETWEEN THE DIFFERENT APPROACHES

To overcome the time issue, the authors of [1] and [15] introduced the hierarchical approach for the PLL network performance analysis with two sub-system characterizations: disc and band QCs. The results of the disc [1] and of the disc+band [15] characterizations are presented in Fig. 7. It is clear that the performance analysis is much faster but also more conservative. This paper introduces a new QC: cone sector which considers the phase information. The cone sector is used in combination with the disc QC obtained in [1]. Namely, in the PLL application example, the cone sector offset is fixed as $C=c_{d i s c}+i r_{d i s c}$ where $c_{d i s c}$ and $r_{d i s c}$ are the disc centre and radius respectively obtained from the disc QC [1]. It is clear that this combination is more suitable for the PLL network where the obtained result is almost the same as the result of direct $\mu$-analysis approach but in much less time, see Fig. 7.

In a general way, the conservatism of the hierarchical approach can be reduced by combining multiple dissipativity properties from the local step: disc+bands+cones. However, to obtain precise result it is better to combine the dissipativity properties of different nature. Of course, the computation time will increase with the number of dissipativity properties used, however since they are used in local step, and thus potentially for a system of small dimensions (local subsystem), this increase is limited within a reasonable time. The contribution of this paper, is an introduction of a new QCs that take into account the information on uncertain subsystem phase. One can thus better define a trade-off between conservatism and efficiency.

\section{CONCLUSION}

In this paper, the performance analysis of uncertain large scale systems is considered. In order to reduce the complexity and the computation time, the hierarchical approach is used to investigate the performance of a PLL network. A new IQC is proposed for the local step which is the phase uncertainty characterization. SISO and MIMO examples are used to illustrate the efficiency of this characterization. This new IQC confirmed its advantage in obtaining less conservative results in the hierarchical approach comparing with the already existing IQCs in literature. For the future work, it would be interesting to investigate the appropriate decomposition of the network into sub-networks in order to have the less conservative results in a reasonable time i.e. add an additional indicator defining conservatism/computation time trade-off.

\section{REFERENCES}

[1] M. Dinh, A. Korniienko, and G. Scorletti, "Embedding of uncertainty propagation: application to hierarchical performance analysis," IFAC Symposium on System, Structure and Control, vol. 5, no. 1, pp. 190195, 2013.

[2] A. Korniienko, G. Scorletti, E. Colinet, E. Blanco, J. Juillard, and D. Galayko., "Control law synthesis for distributed multi-agent systems: Application to active clock distribution networks," IEEE American Control Conference, pp. 4691-4696, 2011.

[3] A. Korniienko, G. Scorletti, E. Colinet, and E. Blanco, "Performance control for interconnection of identical systems: Application to PLL network design," International Journal of Robust and Nonlinear Control, no. DOI: 10.1002/rnc.3285, 2014.

[4] S. Boyd and L. Vandenberghe, Convex Optimization. Cambridge University Press, 2004. 
[5] M. K. H. Fan, A. L. Tits, and J. C. Doyle, "Robustness in the presence of mixed parametric uncertainty and unmodeled dynamics," IEEE Transactions on Automatic Control, vol. 63, pp. 25-38, Jan. 1991.

[6] J. Doyle, "Analysis of feedback systems with structured uncertainties," IEEE Proceddings, vol. 129-D, p. 242:250, nov 1982.

[7] A. Megretski and A. Rantzer, "System analysis via integral quadratic constraints," IEEE Transaction On Automatic Control, vol. 42, jun 1997.

[8] C. Y. Kao, U. Jönsson, and H. Fujioka, "Characterization of robust stability of a class of interconnected systems," Automatica, vol. 45, no. 1, pp. 217-224, 2009.

[9] U. Jönsson, C. Y. Kao, and H. Fujioka, "A popov criterion for networked systems," Systems and Control Letters, vol. 56, pp. 603610, 2007.

[10] U. Jönsson and C. Y. Kao, "A scalable robust stability criterion for systems with heterogeneous LTI components," IEEE Transactions on Automatic Control, vol. 55, no. 10, pp. 2219- 2234, 2010.

[11] I. Letas and G. Vinnicombe, "Scalabe decentralized robust stability certificates for networks of interconnected heterogenous dynamical systems," IEEE Transaction on Automatic Control, vol. 51, pp. 16131626 , oct 2006.

[12] M. Anderson, A. Hanson, S. Pakazad, and A. Rantzer, "Distributed robust stability analysis of interconnected uncertain systetems," IEEE Conference on Decision and Control, pp. 1584-1553, dec 2012.

[13] M. Anderson, S. Pakazad, A. Hanson, and A. Rantzer, "Robust stability analysis of sparsely interconnected uncertain systems," IEEE Transactions on Automatic Control, vol. 59, pp. 2151-2156, aug 2014.

[14] J. R. S. Blair and B. W. Peyton, "An introduction to chordal graphs and clique trees," in Graph Theory and Sparse Matrix Computation (J. R. G. J. A. George and J. W. H. Liu, eds.), vol. 56 of The IMA Volumes in Mathematics and its Applications, pp. 1-29, Springer New York, 1993.

[15] M. Dinh, A. Korniienko, and G. Scorletti, "Convex hierrachical analysis for the performance of uncertain large scale systems," IEEE Conference on Decision and Control, pp. 5979- 5984, 2014.

[16] M. G. Safonov, "Propagation of conic model uncertainty in hierarchical systems," IEEE Transactions on Automatic Control, vol. 28 , pp. 701-709, jun 1983.
[17] I. Postlethwaite, J. M. Edmunds, and A. G. J. Macfarlane, "Principa gains and principal phases in the analysis of linear multivariable feedback systems," IEEE Transactions on Automatic Control, vol. 26, pp. 32-46, feb 1981.

[18] D. H. Owens, "The numerical range : A tool for robust stability studies?," Systems and Control Letters, pp. 153-158, 1984.

[19] P. Harshavardhana and E. A. Jonckheere, "Spectral factor reduction by phase matching: the continuous-time single-input single-output case,' International Journal of Control, vol. 42, no. 1, pp. 43-63, 1985.

[20] J. R. Bar-On and E. A. Jonckheere, "Phase margin for multivariable control systems," International Journal of control, vol. 52, no. 2, pp. 485-498, 1990.

[21] A. Ifftar and Ü. Özgüner, "Modeling of uncertain dynamics for robust controller design in state space," Automatica, vol. 27, no. 1, pp. 141$146,1991$.

[22] A. Tits, V. Balakrishnan, and L. Lee, "Robustness under bounded uncertainty with phase information," IEEE Transactions on Automatic Control, vol. 44, pp. 50-65, jan 1999.

[23] V. Chellaboina, W. M. Haddad, and A. Kamath, "The structured phase margin for robust stability analysis of linear systems with phase and time delay uncertainties," International Journal of Control, vol. 81, pp. 1298-1310, aug 2008.

[24] G. Scorletti, "Robustness analysis with time delays," in IEEE Conference on Decision and Control (IEEE, ed.), (San Diego, California), pp. 3824-3829, Dec. 1997

[25] J. Doyle, A. Packard, and K. Zhou, "Review of LFTs, LMIs, and $\mu$," Proceedings of the 30th IEEE Conference on Decision and Control, vol. 2, pp. 1221- 1232, 1991.

[26] S. Boyd, L. E. Ghaoui, E. Feron, and V. Balakrishnan, "Linear matrix inequalites in system and control theory," SIAM Studies in Applied Mathematics, vol. 15, jun 1994.

[27] Y. Nesterov and A. Nemirovski, Interior Point Polynomial Methods in Convex Programming: Theory and Applications. SIAM, 1994.

[28] G. Scorletti, X. Bombois, M. Barenthin, and V. Fromion, "Improved efficient analysis for systems with uncertain parameters," IEEE Conference on Decision and Control, pp. 5038-5043, dec 2007. 